\title{
VELOCITY-SATURATION RELATION TRANSITION DURING ROCKS SATURATION: DIRECT LABORATORY OBSERVATION BY COMPUTER TOMOGRAPHY AND ULTRASONIC TECHNIQUE
}

\author{
Maxim Lebedev ${ }^{1 *}$, Boris Gurevich ${ }^{2}$, Ben Clennell ${ }^{3}$, Marina Pervukhina ${ }^{4}$, \\ Valeria Shulakova ${ }^{5}$, Tobias Müller ${ }^{6}$ \\ Curtin University of Technology m.lebedev@curtin.edu.aul, Curtin University of Technology and \\ CSIROPetroleumb.gurevich@curtin.edu.au',CSIROPetroleumben.clennell@sciro.au,.CSIRO \\ Petroleum marina.pervukhina@csiro.au, CSIROPetroleum,valeria.shuljkova@sciro.aus,CSIRO \\ Petroleum tobias.mueller@csiro.au ${ }^{6}$
}

Key Words: reservoir characterisation, rock properties, patchy saturation, poroelasticity, 4D-monitoring,

\section{INTRODUCTION}

Maximising the recovery of known hydrocarbon reserves is one of the biggest challenges facing the petroleum industry today. Optimal production strategies require accurate monitoring of production-induced changes of reservoir saturation and pressure over the life of the field. Timelapse seismic technology is increasingly used to map these changes in space and time. However until now, interpretation of time-lapse seismic data has been mostly qualitative. In order to allow accurate estimation of the saturation, it is necessary to know the quantitative relationship between fluid saturation and seismic characteristics (elastic moduli, velocity dispersion and attenuation). The problem of calculating acoustic properties of rocks saturated with a mixture of two fluids has attracted considerable interest (Gist, 1994; Mavko and Nolen-Hoeksema, 1994; Knight et al. 1998); for a comprehensive review of theoretical and experimental studies of the patchy saturation problem see Toms et al. (2006).

For a porous rock whose matrix is elastically homogeneous, and inhomogeneities are caused only by spatial variations in fluid properties, two theoretical bounds for the P-velocity are known (Mavko and Mukerji, 1998, Mavko et al., 1998). In the static (or low-frequency) limit, saturation can be considered as homogeneous, and hence the rock may be looked at as saturated with a homogeneous mixture of the fluids. In this case the bulk modulus of the rock is defined by the Gassmann equation with the fluid bulk modulus given by Wood's formula i.e., the saturation-weighted harmonic average of the bulk moduli of fluids. The Gassmann-Wood bound is valid when the characteristic patch size is small compared to the fluid diffusion length. The diffusion length is primarily controlled by rock permeability, fluid viscosity and wave frequency. In the opposite case, when the patch size is much larger than the diffusion length, there is no pressure communication between fluid pockets and consequently no fluid flow occurs. In this no-flow (or high-frequency) limit, the overall rock behaves like an elastic composite consisting of homogeneous patches whose elastic moduli are given by Gassmann's theory. Since all these patches have the same shear modulus, the effective P-wave modulus can be obtained using Hill's equation, i.e., the saturation-weighted harmonic average of the P-wave moduli. Whereas the Gassmann-Wood and Gassmann-Hill bounds apply in the low- and highfrequency limits, respectively, for intermediate frequencies, uneven deformation of fluid patches by the passing wave results in local pressure gradients and hence in the wave-induced fluid flow. Such wave-induced flow causes wave attenuation and thus velocity dispersion. The effects of regularly distributed fluid patches of simple geometry (spheres, flat slabs) were first studied by White (1975), White at al. (1975), and Dutta and Ode (1979). More recently, the effect of regularly distributed patches of more general shape was modelled by Johnson (2001) and Pride et al. (2004). For randomly distributed fluid patches, Müller and Gurevich (2004) and Müller et al. (2008) showed how the effect of wave-induced flow controls the transition from the Gassmann-Wood to the Gassmann-Hill bounds. 
While theoretical poroelastic models can predict the acoustic response for a given spatial distribution of fluid patches, the factors controlling the formation of the patches are less well understood. These factors can be studied using fluid-injection experiments in the laboratory. Previously reported laboratory observations demonstrate a qualitative link between fluid patch distribution and acoustic velocities (Cadoret et al., 1995, 1998; Monsen and Johnstad, 2005). In order to get a deeper insight into the factors influencing the patch distribution and the associated wave response, we perform simultaneous measurements of P-wave velocities and rock sample $\mathrm{X}$-ray computer tomography (CT) imaging. The CT imaging allows us to infer the fluid distribution inside the rock sample during saturation (water imbibition). We then show that the experimental results are consistent with theoretical predictions and numerical simulations.

\section{EXPERIMENTAL SETUP}

Experiments are performed on a cylindrical sample (38 $\mathrm{mm}$ in diameter and $60 \mathrm{~mm}$ long) cut from a Casino sandstone (Otway Basin, Australia). The sample is dried at $100{ }^{\circ} \mathrm{C}$ under reduced pressure for $24 \mathrm{~h}$. The petrophysical properties are measured using a Coretest AP-608 automatic permeameter/porosimeter (Table 1). Then, the sample is sealed with a thin epoxy layer in order to prevent fluid leakage through the surface. Longitudinal $(\mathrm{Vp})$ and shear wave $(\mathrm{Vs})$ velocities at a frequency of $1 \mathrm{MHz}$ are measured in the direction Table 1: Petrophysical properties of the dry rock sample

\begin{tabular}{|l|l|}
\hline \hline & $\begin{array}{l}\text { Casino } \\
\text { Otway }\end{array}$ \\
\hline Bulk density, g/cm ${ }^{3}$ & 2.2 \\
\hline Grain density, $\mathrm{g} / \mathrm{cm}^{3}$ & 2.65 \\
\hline Porosity, \% & 16.7 \\
\hline Permeability, $\mathrm{mD}$ & 7.26 \\
\hline
\end{tabular}
across to the core axis (perpendicular to the fluid flow) using broadband ultrasonic transducers. Intermediate aluminum "guide-pins" are placed between the sample and transducers to secure sufficient and constant coupling, as well as to provide transparency for X-ray radiation.

Two different saturation methods - referred to as dynamic and quasi-static saturation - are used in this study. In the dynamic saturation experiments, the samples are jacketed in the experimental cell made out of X-ray transparent material PMMA (Figure 1). Distilled water is injected into the sample from one side. The injection rate is $10 \mathrm{ml}$ per day. In quasi-static saturation experiments the samples are saturated during a long period of time (up to 2 weeks) under reduced pressure in order to achieve near-uniform fluid distribution for a given saturation level. For quasi-static experiments the saturation level is determined by measurement of the volume (weight) of water fraction divided by total volume of pores. A less than $1 \%$ difference
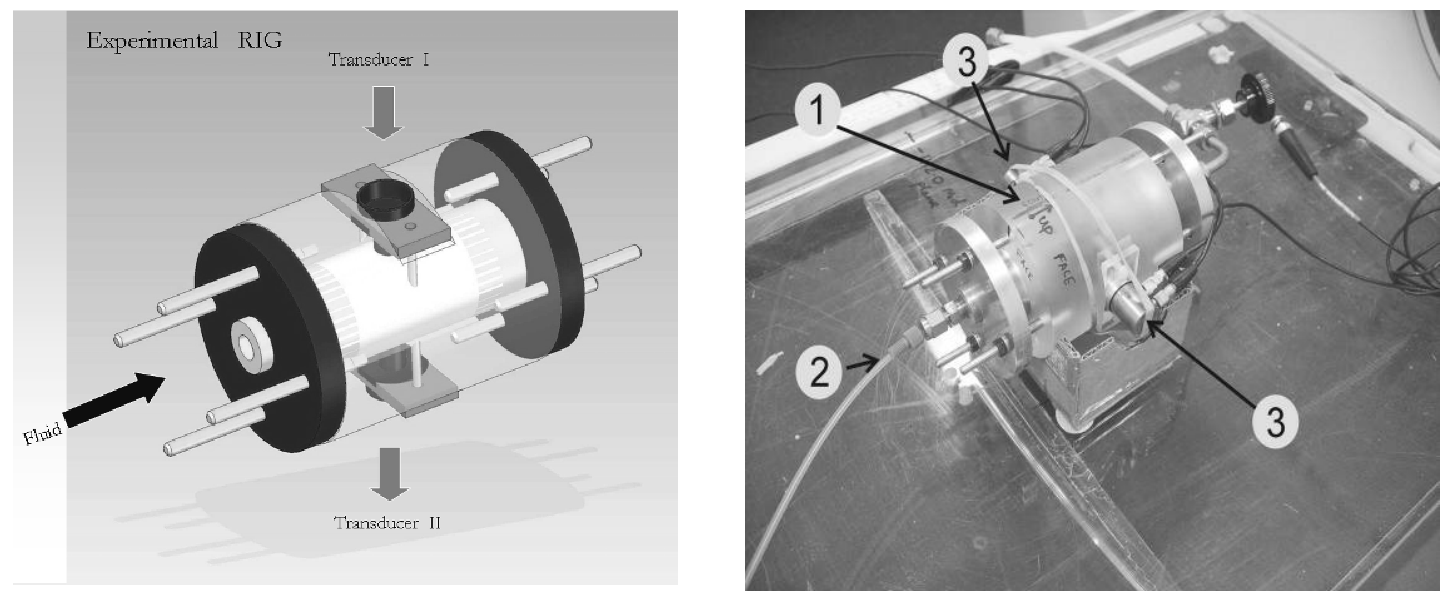

Figure 1: Core sample jacketed inside experimental rig: 1- X-ray transparent jacket; 2 injection pipe; 3 - ultrasonic transdusers. 
in velocities measured in different directions indicates that fluid is uniformly distributed within the sample.

The fluid distribution in the dynamic saturation experiments (both spatial and time dependence) is characterised using $\mathrm{X}$-ray computer tomography. (Figure 2). The resolution is $0.2 \times 0.2 \times 1 \mathrm{~mm} 3$ (voxel size). This resolution is not sufficient to image the exact fluid patch geometry; however, it shows overall character of the fluid distribution. The fluid saturation is estimated as a difference in average CT-number (a value related to material density) between the saturated and dried sample divided by the volume of porous fraction. The temporal evolution of the saturation profile along the rock sample is shown in Figure 3. We see that the overall saturation profile becomes progressively more uniform over time. All

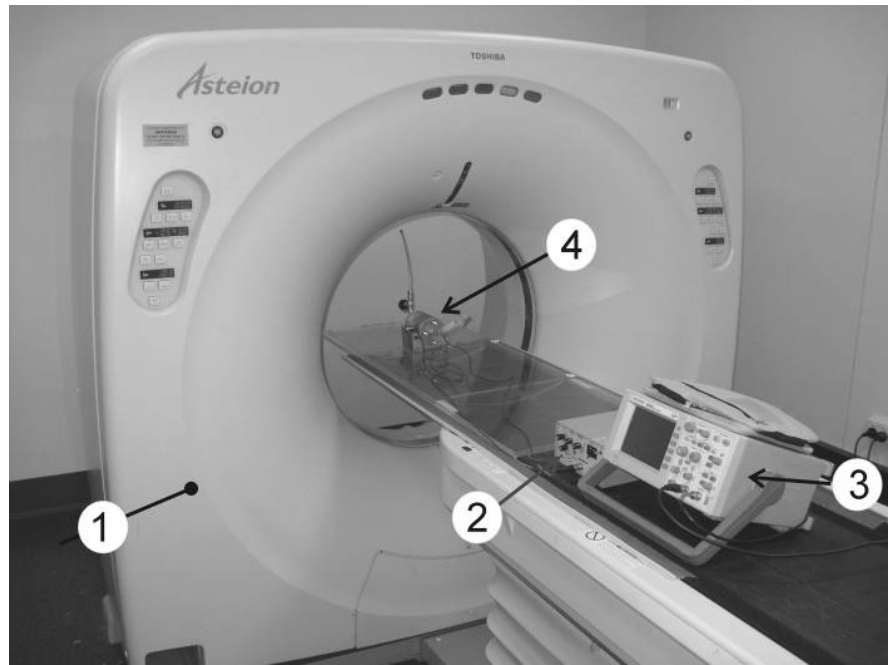

Figure 2: Simultanious aquisition of acoustic properties and fluid saturation using ultrasonic transducrs and CT scan: 1 - Computer tomograph; 2 - ultrasonic pulsereceiver; 3 - oscilloscope; 4- jacketed sample experiments are performed in a laboratory environment at a temperature of $25^{\circ} \mathrm{C}$.

\section{EXPERIMENTAL RESULTS}

Velocity is obtained by first break picking on output signal. Figure 4 shows the Pwave velocity as a function of saturation for the Casino Otway sandstone from the dynamic saturation experiment (empty symbols) and quasi-static saturation experiment (solid symbols). A transition from the Gassmann-Wood to Gassmann-Hill bound is clearly observed for water saturations of 40 $50 \%$ for dynamic saturation and $60-70 \%$ for quasi-static saturation. Figure 5 shows CT images recorded during the dynamic saturation experiments. Scans 1-5 correspond to points $1-5$ on the velocitysaturation curve (Figure 4): increasing saturation from the dried sample (1) up to $50 \%$ (point 4) and then decreasing saturation down to $26 \%$ (point 5). At low saturations, the velocity can be well described by the Gassmann-Wood relation; however, a sharp increase in velocity is observed when saturation

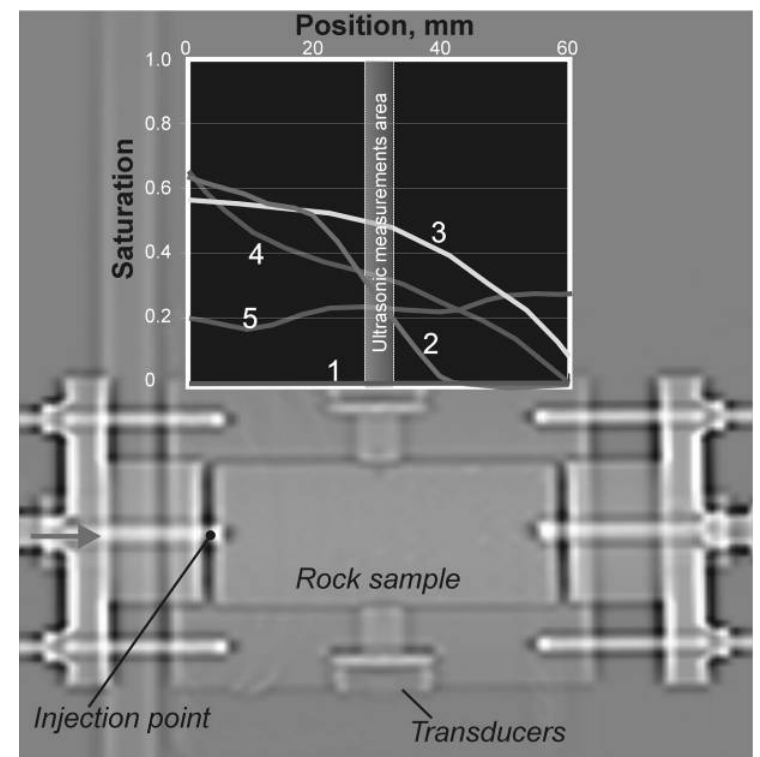

Figure 3: X-ray image of the whole core sample. The embedded plot shows the profiles of water saturation along the $60 \mathrm{~mm}$ long sample at differnet times (increasig from 1 to 5) during the saturation experiment. 
exceeds $40 \%$ (points 3 and 4 in the Figure 4). Then, the velocity-saturation approaches the Gassmann-Hill bound.

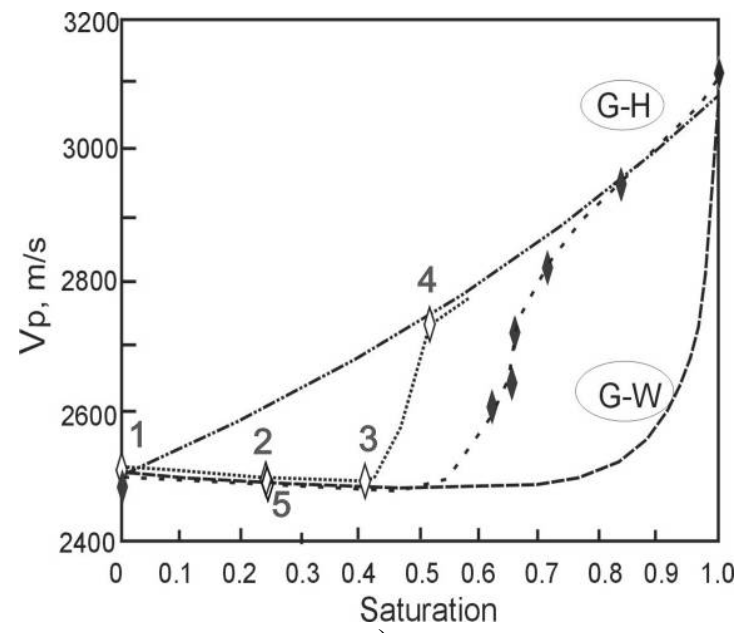

a)

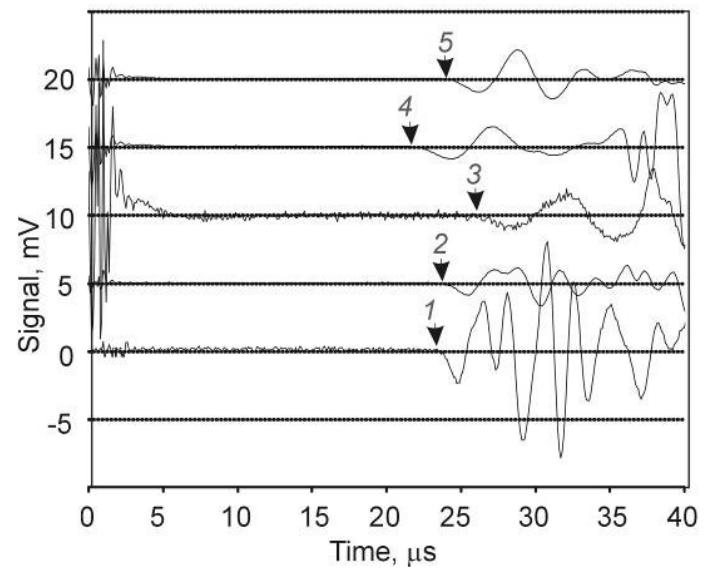

b)

Figure 4: a) Velocity versus saturation for the Casino Otway Basin sandstone from the quasistatic saturation experiment (blackdiamonds) and dynamic saturation experiment (white diamonds). The numbers from 1 to 5 indicate acoustic measurements corresponding to the CT images shown in Figure 5, and saturation profiles shown in Figure 3. Theoretical Gassmann-Wood $(G-W)$ and Gassmann-Hill $(G-H)$ bounds are shown by dashed and dashdotted lines, respectively. b) Output signals corresponding to five stages during the dynamic fluid injection experiment.

We also observe that for relatively large fluid injection rates (dynamic experiment) the transition from homogeneous to patchy saturation (from the Gassmann-Wood to GassmannHill) occurs at smaller degrees of saturation as compared with low injection rates (quasi-static experiment). This observation suggests that the process of fluid patch formation is controlled by the injection rate.

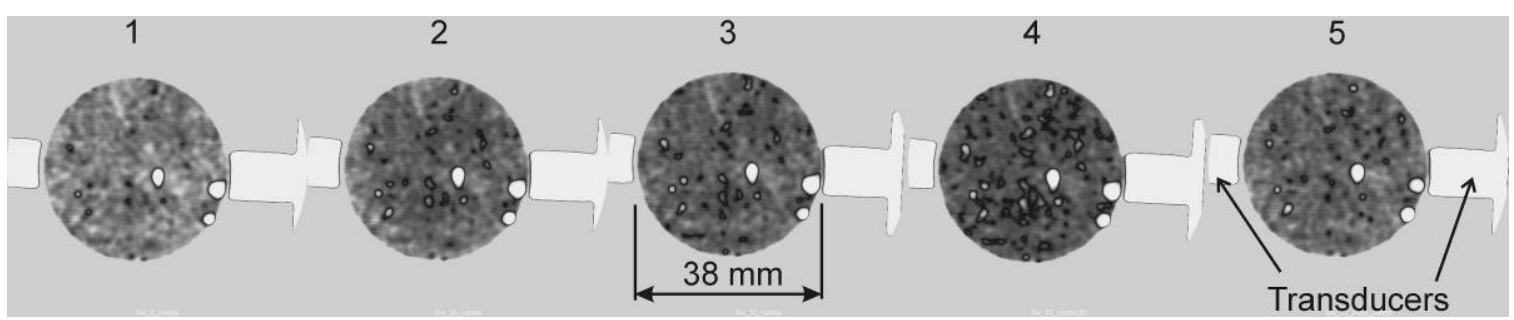

Figure 5: CT images of the Casino Otway Basin sandstone at different times after start of injection at the same position: 1 -dried sample; $2-1 \mathrm{~h} ; 3-24 \mathrm{~h} ; 4-72 \mathrm{~h}$. Contour of guidepins are visible at right- and left-hand side of the sample. 


\section{NUMERICAL SIMULATIONS}

In order to validate our interpretation of the velocitysaturation relation in terms of wave-induced flow we also perform numerical simulations of wave propagation in a 2-D poroelastic solid. The simulations are performed using 2D finite-difference solver of Biot's equations of dynamic poroelasticity (Krzikalla and Müller, 2007). Figure 6 shows the modelled velocity-saturation relation obtained for material constants and wave frequency used in laboratory experiment described above. The degree of saturation is varied by increasing the size of the randomly distributed fluid patches. It can be seen that for large saturations, the fluid

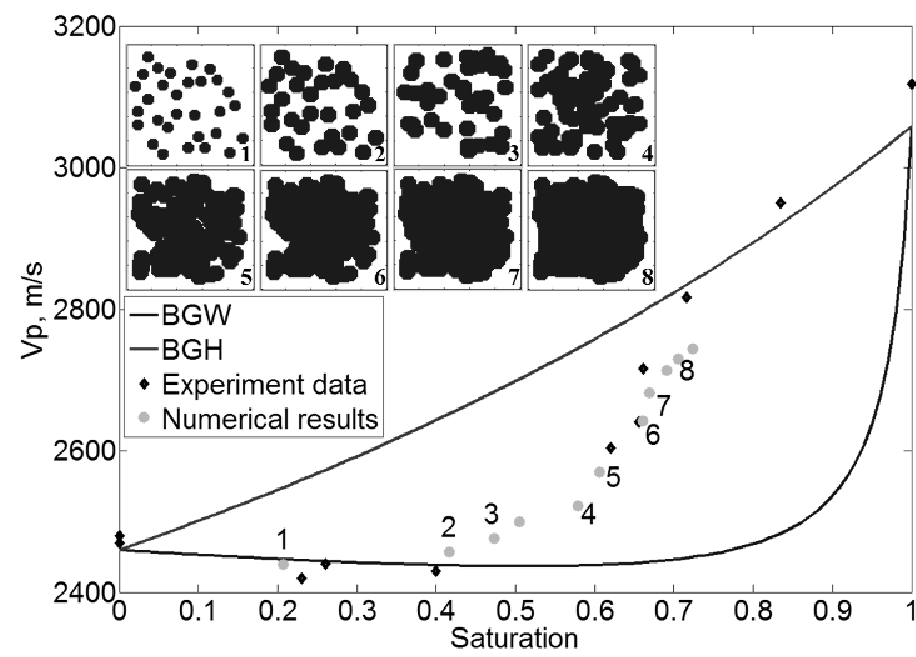

Figure 6: Velocity-saturation relation determined from numerical simulations of wave propagation in a poroelastic solid with randomly distributed patches that cluster for larger saturation values (see inset). Experimentally determined velocities for the quasi-static injection experiment are also shown. patches begin to cluster resembling the clustering observed in the CT scans. Despite the simplified numerical setup (2D modelling only and patch distribution not derived directly from the CT scans) the simulation results reproduce the overall behaviour of the measured velocity-saturation relation. This confirms that the transition observed in the ultrasonic measurements can be attributed to the mechanism of wave-induced flow. We plan to extend our numerical simulations to 3D so that the 3D patch distributions reconstructed from CT images can be directly used as input model in our simulations.

\section{CONCLUSIONS}

The experimental results obtained on low-permeability samples show that at low saturation values the velocity-saturation dependence can be described by the Gassmann-Wood relationship. At intermediate saturations there is a transition behaviour that is controlled by the fluid patch arrangement and fluid patch size. Also, the fluid patch size is controlled by the injection rate. In particular, we show that for relatively large fluid injection rate this transition occurs at smaller degrees of saturation as compared with high injection rate.

The results illustrate the non-unique relationships between saturation and velocity in sandstones dependent on texture and fluid displacement history: fuller understanding of these phenomena are needed for accurate assessment of time lapse seismic measurements, be they for oil and gas recovery or for $\mathrm{CO}_{2}$ disposal purposes

\section{REFERENCES}

Cadoret, T., D. Marion, and B. Zinszner, 1995, Influence of frequency and fluid distribution on elastic wave velocities in partially saturated limestones: Journal of Geophysical Research, 100, 9789-9803. 
Cadoret, T., G. Mavko, and B. Zinszner, 1998, Fluid distribution effect on sonic attenuation in partially saturated limestones: Geophysics, 63, 154-160.

Dutta, N. C., and Odé, H., 1979, Attenuation and dispersion of compressional waves in fluidfilled porous rocks with partial gas saturation (White model) - Part I: Biot theory: Geophysics $44,1777-1788$.

Gassman, F., 1951, Elastic waves through a packing of spheres. Geophysics 16, 673-685

Gist, A.G., 1994, Interpreting laboratory velocity measurements in partially gas-saturated rocks: Geophysics, 54, 1100-1108.

Johnson, D. L., 2001, Theory of frequency dependent acoustics in patchy-saturated porous media: Journal of the Acoustical Society America, 110, 682- 694.

Knight, R., J. Dvorkin, and A. Nur, 1998, Acoustic signatures of partial saturation: Geophysics, $63,132-138$.

Krzikalla, F., Müller, T.M., 2007, High-contrast finite-differences modeling in heterogeneous poroelastic media: 77th SEG Annual Meeting, San Antonio, Extended Abstracts, 2030-2034.

Mavko, G., Nolen-Hoeksema, R., 1994, Estimating seismic velocities at ultrasonic frequencies in partially saturated rocks: Geophysics, 59, 252-258.

Mavko, G. and T. Mukerji, 1998, Bounds on low frequency seismic velocities in partially saturated rocks: Geophysics, 63, 918-924.

Mavko, G., Mukerji, T., and Dvorkin, J., 1998, The Rock Physics Handbook: Tools for seismic analysis in porous media: Cambridge University Press.

Monsen, K. and S. E. Johnstad, 2005, Improved understanding of velocity-saturation relationships using 4D computer-tomography acoustic measurements: Geophysical Prospecting, 53, 173-181.

Müller, T. M. and B. Gurevich, 2004, One-dimensional random patchy saturation model for velocity and attenuation in porous rocks: Geophysics, 69, 1166-1172.

Müller, T. M.; Toms-Stewart, J.; Wenzlau, F., 2008, Velocity-saturation relation for partially saturated rocks with fractal pore fluid distribution: Geophysical Research Letters, 35, L09306.

Pride, S.R.,. Berryman, J.G. and Harris, J.M., Seismic attenuation due to wave- induced flow: Journal of Geophysical Research 109 (2004), B01201 1-19.

Toms, J., T. M. Müller, R. Ciz, and B. Gurevich, 2006, Comparative review of theoretical models for elastic wave attenuation and dispersion in partially saturated rocks: Soil Dynamics and Earthquake Engineering, 26, 548-565.

White J. E , 1975, Computed seismic speeds and attenuation in rocks with partial gas saturation: Geophysics 40, 224-232.

White, J. E., N. G. Mikhaylova, and F. M. Lyakhovitskiy, 1976, Low-frequency seismic waves in fluid-saturated layered rocks: Physics of the Solid Earth Transactions. Izvestiya, 11, 654-659. 